\title{
Cholesteryl Hemisuccinate Is Not a Good Replacement for Cholesterol in Lipid Nanodiscs
}

\section{Augustyn, Bozena}

2019-11-21

Augustyn , B , Stepien , P , Poojari , C, Mobarak, E, Polit , A, Wisniewska-Becker , A \& Rog , T 2019 , ' Cholesteryl Hemisuccinate Is Not a Good Replacement for Cholesterol in Lipid Nanodiscs ' , Journal of Physical Chemistry B , vol. 123 , no. 46 , pp. 9839-9845 . https://doi.org/10.1021/acs.jp

http://hdl.handle.net/10138/326559

https://doi.org/10.1021/acs.jpcb.9b07853

unspecified

acceptedVersion

Downloaded from Helda, University of Helsinki institutional repository.

This is an electronic reprint of the original article.

This reprint may differ from the original in pagination and typographic detail.

Please cite the original version. 


\title{
Cholesteryl Hemisuccinate Is Not a Good Replacement for Cholesterol in Lipid Nanodiscs
}

\author{
Bozena Augustyn ${ }^{1,2}$, Piotr Stepien ${ }^{1,3}$, Chetan Poojari, ${ }^{4,5}$, Edouard Mobarak ${ }^{4}$, \\ Agnieszka Polit ${ }^{6}$, Anna Wisniewska-Becker ${ }^{1 *}$, Tomasz Róg ${ }^{4 *}$
}

\footnotetext{
${ }^{1}$ Department of Biophysics, Faculty of Biochemistry, Biophysics and Biotechnology, Jagiellonian University, Gronostajowa 7, 30-387, Kraków, Poland.

${ }^{2}$ Ardigen, Podole 76, 30-394, Krakow, Poland.

${ }^{3}$ Bionanoscience and Biochemistry Laboratory, Malopolska Centre of Biotechnology, Jagiellonian University, Gronostajowa 7A, 30-387 Krakow, Poland.

${ }^{4}$ Department of Physics, Faculty of Science, University of Helsinki, P.O. Box 64, FI-00014 Helsinki, Finland.

${ }^{5}$ Theoretical Physics and Center for Biophysics, Saarland University, 66123 Saarbrücken, Germany.

${ }^{6}$ Department of Physical Biochemistry, Faculty of Biochemistry, Biophysics and Biotechnology, Jagiellonian University, Gronostajowa 7, 30-387, Kraków, Poland.
}

Corresponding authors: Anna Wisniewska-Becker: anna.m.wisniewska@uj.edu.pl; Tomasz Róg: tomasz.rog@helsinki.fi. 


\section{Abstract}

Nanodiscs are suitable tool for studies of membrane proteins (MPs) due to their ability to mimic native biological membranes and several MP structures are solved in nanodiscs. Among the various cell membrane components, cholesterol ( $\mathrm{CHL}$ ) is known to regulate protein function and their concentration can reach up to $50 \mathrm{~mol} \%$. However, studies comprising cholesterol is challenging due to its hydrophobic nature, hence, nanodiscs with only low cholesterol concentration has been studied. To overcome the problem, cholesterol analogs with high solubility in polar solutions are often used and one of them is cholesteryl hemisuccinate (CHS). Nevertheless, in molecular dynamics (MD) simulation, this is not an obstacle. In this study, we performed MD simulations of nanodiscs containing neutral phosphatidylcholine (POPC) lipids, negatively charged phosphatidylglycerol (POPG) lipids, $\mathrm{CHL}$, or negatively charged cholesterol analog, CHS. Our simulations show that CHS increases the order of lipids in nanodiscs, the effect is however weaker than $\mathrm{CHL}$, and even smaller in nanodiscs. Furthermore, CHS gathered around scaffold proteins while cholesterol was uniformly distributed in the nanodiscs. Thus, nanodiscs with $\mathrm{CHS}$ are heterogeneous and not equivalent to nanodiscs with CHL. Finally, we also observed the increased concentration of POPG near the scaffold proteins, driven by electrostatic interactions. The MD results are experimentally validated using electron paramagnetic resonance spectroscopy. These results show that nanodiscs are in fact, complex structures not easily comparable with planar lipid bilayers. 


\section{Introduction}

Nanodiscs are nanoscale macromolecules, composed of lipid bilayer wrapped with two membrane scaffold proteins (MSPs). Nanodiscs are engineered based on apolipoprotein ApoA I, a component of high-density lipoprotein. ${ }^{1-3}$ Primary reason for the development of nanodiscs are challenges related to studies of membrane proteins (MPs), ${ }^{4}$ which require hydrophobic environment naturally ensured by lipids. Use of nanodiscs as a platform to study MPs seems to be an excellent alternative to commonly used micelles, bicelles, and liposomes, ${ }^{5}$ since all of them have some disadvantages. ${ }^{6}$ Micelles do not have the bilayer structure, bicelles have bilayer like arrangement, but they require high concentration of lipids and detergents. Liposomes are the most suitable systems in structural investigations; however, they are large, unstable, and challenging to prepare.

Nanodisc technology is a flexible method allowing modification of properties of nanodiscs and optimizing them for the desired application. For instance, by changing the length of MSPs, it is possible to obtain nanodiscs of a precisely defined size in the range from $6 \mathrm{~nm}$ to $16 \mathrm{~nm}$ and well-defined number of lipids. ${ }^{2,7,8}$ Moreover, the lipid composition of nanodisc can be altered to increase the nanodisc stability. ${ }^{9}$ Lipid composition is of particular importance for MPs as they are inseparable in the lipid environment and can affect the structure, activity, and dynamics of MPs. In this respect, cholesterol $(\mathrm{CHL})$ is of particular importance in animal membranes. ${ }^{10,11} \mathrm{CHL}$ occurs in large quantities in cell membranes with concentration reaching about 50 mol\%, but in intracellular membranes e.g., in endoplasmic reticulum the concentration is only about $10 \mathrm{~mol} \% .^{12,13} \mathrm{CHL}$ regulates not only the physicochemical properties of lipid bilayers like bilayer thickness, rigidity, polarity, and permeability but also known to be a direct allosteric regulator of MPs. ${ }^{14-18}$ Nevertheless, due to its highly hydrophobic nature, it is often not possible to use $\mathrm{CHL}$ in biochemical studies. ${ }^{19}$ For this reason, nanodiscs with only low $\mathrm{CHL}$ concentrations have been successfully fabricated. ${ }^{20,21} \mathrm{As}$ an alternative, $\mathrm{CHL}$ structural analogs with high solubility in polar solutions are often used and one of them is cholesteryl hemisuccinate (CHS). ${ }^{19}$

CHS is an ester of cholesterol and succinic acid, and due to the presence of the carboxylic group, it has characteristics of typical detergent (Figure 1). Of late, CHS has been frequently added detergent in the crystallization of MPs, particularly GPCRs. ${ }^{22-24}$ Thus, the presence of 
CHS in $12 \mathrm{MP}$ structures is not surprising. ${ }^{25}$ The position occupied by $\mathrm{CHS}$ in these protein structures is believed to be the same for $\mathrm{CHL}$ in natural bilayers. $\mathrm{CHS}$ is also used to stabilize MPs structure in biochemical experiments utilizing micelles or bicelles. ${ }^{23,26-30}$ Since $\mathrm{CHL}$ and CHS share the same hydrophobic section of the molecule, these sterols are considered to be equivalent in biochemical studies. This assumption is however questionable based on the biophysical studies of sterol induced changes of lipid bilayer properties. ${ }^{14}$ The studies showed that even small structural modifications of native cholesterol structure decrease the ability of the sterol to modify lipid bilayer properties. ${ }^{14} \mathrm{CHS}$ is no exception here, as most sterols it increases the order of the lipid acyl tails and stabilizes the bilayer but these effects are weaker when compared to $\mathrm{CHL} .{ }^{31,32}$ The CHS carboxylic group has pKa of 5.8; thus approximately $98 \%$ of the CHS present at the physiological $\mathrm{pH} 7$ will be present in the anionic form. ${ }^{33}$ Consequently, the presence of CHS causes an effective change of the surface charge of the bilayer.

a) $\mathrm{POPC}$

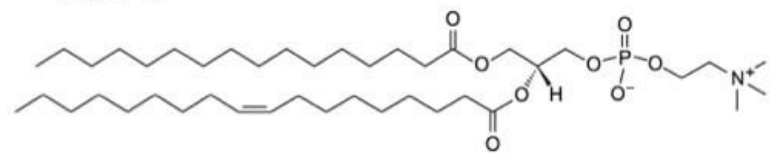

POPG
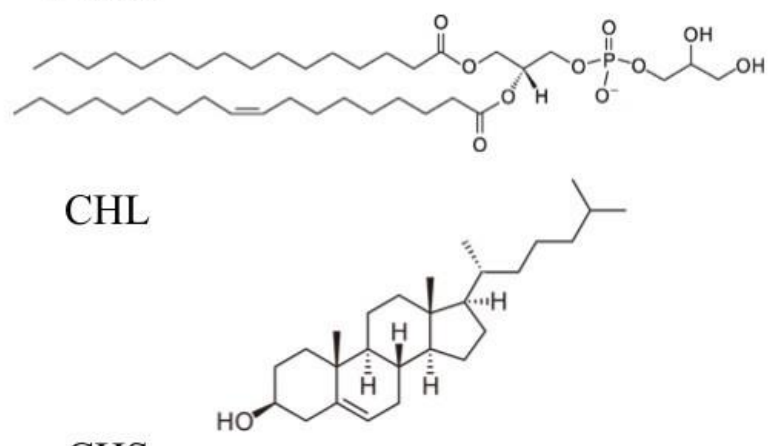

CHS
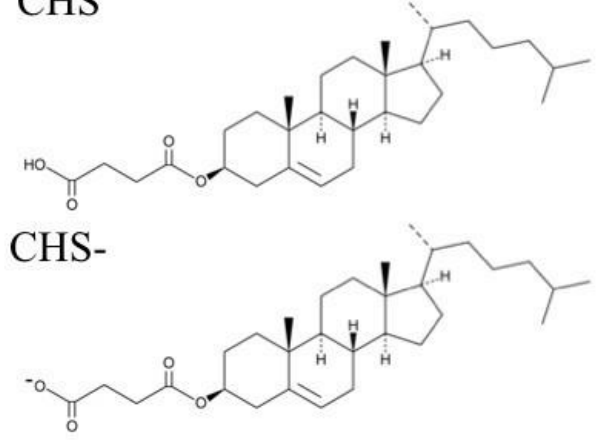

b)
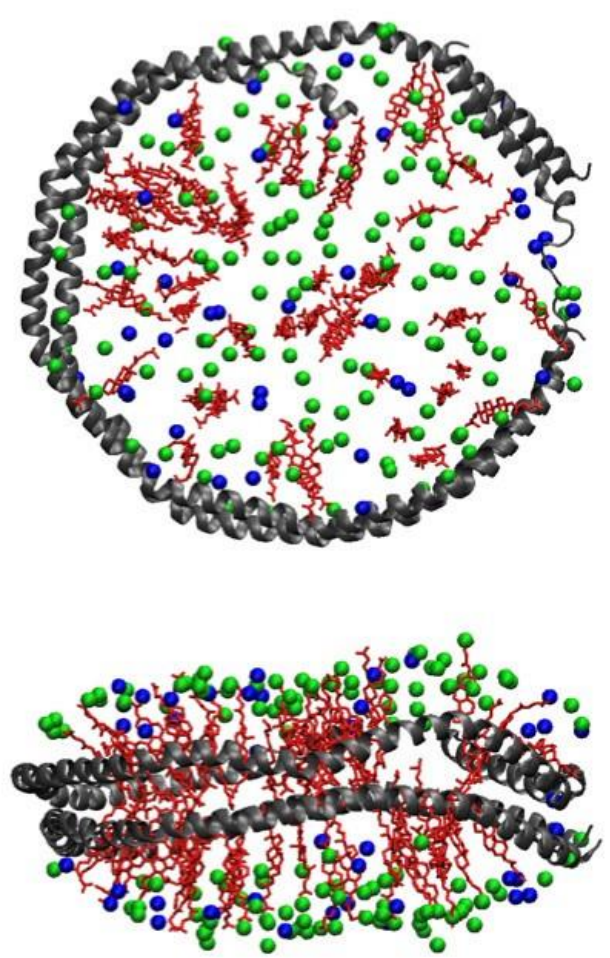

Figure 1. Figure showing (a) chemical structures of lipid molecules and (b) initial configuration of POPC/POPG/CHL nanodiscs. MSP1D1 is rendered as gray cartoon, $\mathrm{CHL}$ as red licorice, phosphorous atoms of POPC and POPG are rendered as VDW spheres and colored pink and 
purple, respectively.

The main objective of this study is to compare the effects of CHS and CHL on the properties of lipid bilayer in nanodiscs which we do by combining molecular dynamics (MD) simulations and electron paramagnetic resonance (EPR) spectroscopy. In addition, to compare sterol effects on nanodiscs with more conventional membrane models, lipid bilayer and liposomes were used in MD simulations and EPR measurements, respectively.

\section{MATERIALS AND Methods}

\section{Molecular Dynamics Simulations}

CHARMM-GUI was used to prepare the initial structures of nanodiscs and planar lipid bilayers $^{34-36}$ for MD simulations. Nanodiscs were composed of MSP1D1 protein $(9.7 \mathrm{~nm})$, palmitoyl-oleoyl-phosphatidylcholine (POPC) / palmitoyl-oleoyl-phosphatidylglycerol (POPG) in ratio 3:1, and 25 mol\% of sterols ( $\mathrm{CHL}$ or $\mathrm{CHS}$ ). Figure 1 shows the structure of molecules used in this work and snapshots of initial structure of nanodiscs and planar lipid bilayers. All systems were solvated using TIP3 water, ${ }^{37}$ and $\mathrm{Na}^{+}$and $\mathrm{Cl}^{-}$ions were added to ensure physiological salt concentration and neutrality of the systems. CHS was studied in both neutral ( $\mathrm{CHSO}$ ) and deprotonated form ( $\left.\mathrm{CHS}^{-}\right)$. The exact composition of each system is given in Table 1.

Charmm36 force field ${ }^{38,37}$ was used to describe the protein, lipids and water/ions. Prior to MD simulations, all the systems were energy minimized using steepest-descent algorithm. The equilibration was performed with position restraints applied to the lipid and protein atoms.

Table 1. Numbers of lipids, water, and ions in planar lipid bilayers and nanodiscs used in MD simulations.

\begin{tabular}{|l|l|l|l|l|l|l|}
\hline & POPC & POPG & Sterol & Water & Na+ & Cl- \\
\hline \multicolumn{7}{|c|}{ Planar Lipid Bilayers } \\
\hline POPC/POPG & 96 & 32 & - & 6180 & 44 & 12 \\
\hline POPC/POPG/CHL & 72 & 24 & 32 & 5769 & 35 & 11 \\
\hline POPG/POPG/CHSO & 72 & 24 & 32 & 6917 & 36 & 12 \\
\hline POPG/POPG/CHS & 72 & 24 & 32 & 7050 & 69 & 13 \\
\hline \multicolumn{7}{|l|}{ Nanodiscs } \\
\hline POPC/POPG & 132 & 44 & - & 122565 & 276 & 222 \\
\hline POPC/POPG/CHL & 126 & 42 & 56 & 95337 & 225 & 173 \\
\hline POPC/POPG/CHSO & 126 & 42 & 56 & 120501 & 270 & 218 \\
\hline
\end{tabular}




\begin{tabular}{|l|l|l|l|l|l|l|}
\hline POPC/POPG/CHS & 126 & 42 & 56 & 123247 & 331 & 223 \\
\hline
\end{tabular}

For the long-range electrostatic interactions, the Particle-Mesh Ewald (PME) method was applied with a Coulomb cut-off of $1.2 \mathrm{~nm}$. Lennard-Jones interactions were truncated at the distance of $1.2 \mathrm{~nm} \cdot{ }^{39}$ To preserve the covalent bond lengths the linear constraint solver (LINCS) algorithm was employed, ${ }^{40}$ and the time step of simulations was set to $2 \mathrm{fs}$. The neighbor search was performed using the Verlet cut-off scheme. The neighbor list was updated every 20 steps. Temperature, $310 \mathrm{~K}$, was maintained with Nose-Hoover thermostat, separately for protein, lipids, and water/ions. ${ }^{41,42}$ Pressure, 1 bar, was coupled with Parrinello-Rahman barostat. $^{43}$ The time constant for thermostat was set to 1 ps and for barostat 5 ps. All restraints were removed for the final production run and simulations were carried out using GROMACS 5 package. ${ }^{44}$ The nanodisc system were simulated for $1 \mu \mathrm{s}$ each and planar lipid bilayers for $500 \mathrm{~ns}$ each. All analysis were performed using gromacs tools and two dimensional maps were calculated using g_thickness, g_mydensity, g_ordercg scripts ${ }^{45}$. Figures were rendered using $\mathrm{VMD}^{46}$ tool. The stability of the nanodiscs structure during MD simulations was monitored via evaluation of the secondary structure of the MSP. Both the secondary structure and the disc shape were preserved during simulations. Besides, we monitored the radius of gyration of nanodiscs, which showed that the size of nanodiscs was not changing during simulations. The radius of gyration was the same for CHL and CHSO nanodiscs, $4.84 \pm 0.03,4.83 \pm 0.03$ respectively, and $1 \%$ larger for CHS- nanodisc, $4.90 \pm 0.03$. The shape of nanodiscs was also preserved during MD simulations.

\section{Electron Paramagnetic Spectroscopy and Spectra Simulations}

POPC and POPG lipids were purchased from NOF Europe, and the PC spin labels, CHL and CHS from Avanti Polar Lipids. The Plasmids for MSPs expression were purchased from Addgene ${ }^{4,47}$. All other necessary compounds for protein purification and buffer preparation were purchased from Sigma Aldrich Europe. Both the liposome and nanodisc samples (constisting of POPC/POPG 3:1 or POPC/POPG/CHS 12:3:4) containing $1 \mathrm{~mol} \%$ of lipid spin label for the EPR measurement were prepared and checked for the quality as described in the previous work. ${ }^{48}$ Three spin labels $(5,10$ and 16-PC) with a nitroxide free radical moiety attached to different carbon atoms along the lipid alkyl chain were employed. The EPR measurements were conducted using the 
Bruker EMX spectrometer equipped with EMX ER 4141 VT temperature control unit. Samples were placed in the gas-permeable methylpentene plastic, TPX capillary (i.d. $0.6 \mathrm{~mm}$ ) and deoxygenated inside the resonator using nitrogen fumes from the temperature control unit. All samples were measured at $310 \mathrm{~K}$.

Nonlinear least squares (NLLS) fitting procedures were performed using the program by Budil et al, ${ }^{49}$ which uses the stochastic Liouville equation by Schneider and Freed. ${ }^{50}$ The magnetic parameters of A-tensor used for the spectra simulations were established by measuring the samples at rigid limit temperatures (120 K). Parameters for g-tensor were taken from previous studies. ${ }^{51}$ With fixed magnetic parameters, MOMD (microscopic order and macroscopic disorder) model was employed to perform NLLS fitting to the experimental spectra. ${ }^{52}$ One of the parameters calculated based on EPR spectra simulations was the order parameter $S_{0}$.

\section{RESULTS AND DISCUSSION}

\section{CHS and CHL sterols increase order of lipid bilayers}

First, we evaluated the effect of sterols on the bulk properties of lipids in nanodiscs and planar lipid bilayers. From MD simulations, we calculated the order parameter $S_{C D}$ for sn-1 acyl tails of POPC and POPG according to the formula:

$$
S_{\mathrm{CD}}=\left\langle\frac{3}{2}\left(\cos ^{2} \theta_{i}\right)-\frac{1}{2}\right\rangle
$$

where $\theta_{i}$ is the angle between the C-D bond ( $\mathrm{C}-\mathrm{H}$ in simulations) of the $i$-th carbon atom and the bilayer normal. The $S_{C D}$ profiles (Figure 2) indicate that both sterols increase the order of the acyl chains; however, the effect is weaker in nanodiscs than in planar lipid bilayers. In nanodiscs, $\mathrm{CHL}$ and $\mathrm{CHSO}$ induce similar ordering effect on lipid acyl tails, while the effect of $\mathrm{CHS}^{-}$is weaker. Moreover, $\mathrm{CHS}^{-}$in nanodiscs does not increase the order of the $s n-1$ chain of POPG and a small decrease in the order occurs at the segments located deeper in the membrane (below eight segment). This effect is specific for nanodiscs as in planar lipid bilayers the order of POPG chains increase after addition of sterols to the same level as for POPC acyl chains. 


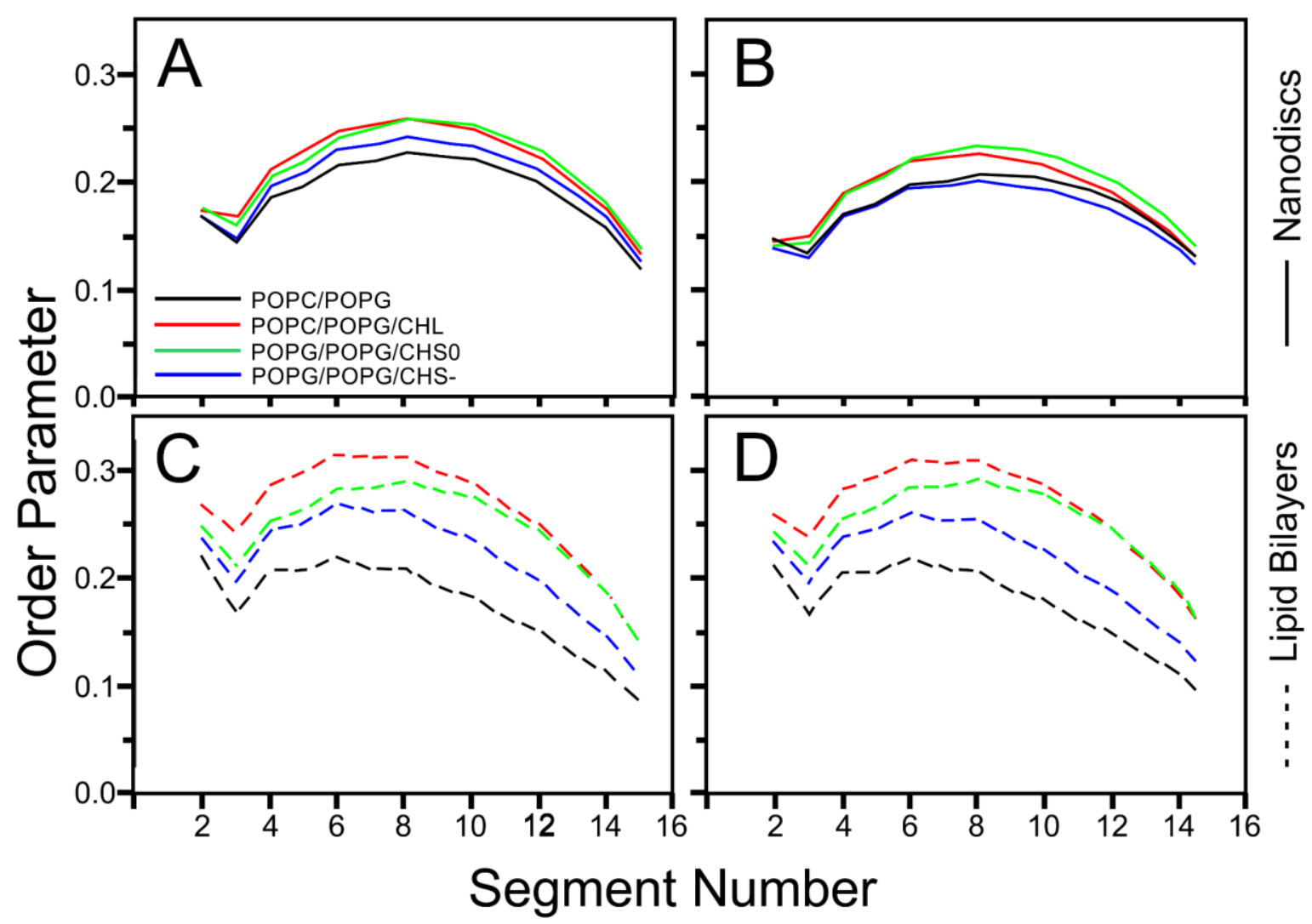

Figure 2. Profiles of the order parameter $S_{C D}$ along sn-1 chain of POPC ( $A$, and $\left.C\right)$, and POPG $(B$, and $D)$ in nanodiscs $(A, B)$, and lipid bilayers (C, and D).

Similarly, from EPR spectroscopy we can obtain $S_{0}$ parameter which is given by the same formula as $S_{C D}$ parameter where $\theta_{i}$ is the angle between bilayer normal and molecular axis of the label..$^{53}$ Profiles of $S_{0}$ parameter for POPC/POPG liposomes in comparison with POPC/POPG and POPC/POPG/CHS nanodiscs are presented in Figure 3a. Profiles of $\mathrm{S}_{0}$ parameter are in qualitative agreement with profiles of $S_{C D}$ parameter and show an increase of the order after addition of CHS to nanodiscs. Additionally, we showed profiles of $\mathrm{S}_{0}$ parameter for POPC/POPG, POPC/POPG/CHL, and POPC/POPG/CHS liposomes (Figure 3b). In agreement with previous studies, the CHS ordering effect is weaker in comparison to CHL. 

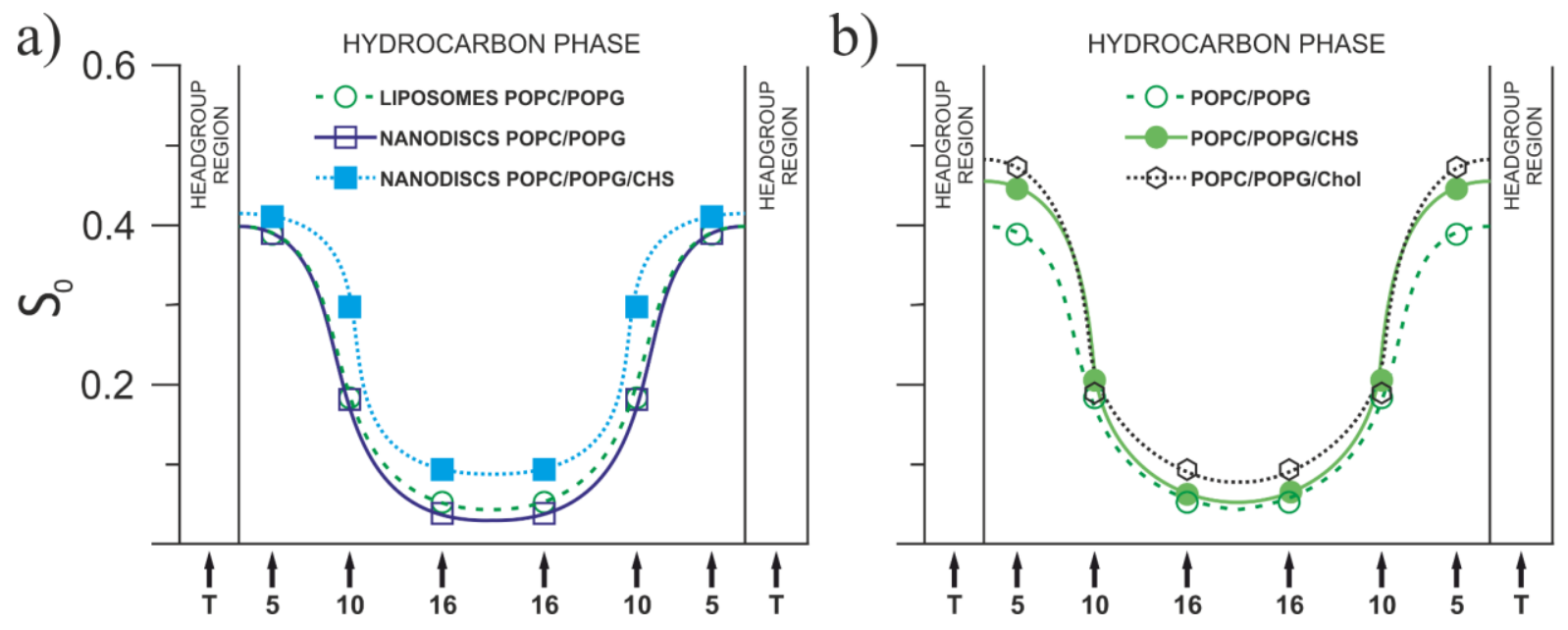

Figure 3. Profiles of the order parameter $S_{0}$ measured in (a) POPC/POPG liposomes, POPC/POPG nanodiscs, and POPC/POPG/CHS nanodiscs; and (b) POPC/POPG liposomes, POPC/POPG/CHL liposomes, and POPC/POPG/CHS liposomes. The approximate locations of the paramagnetic centers in the membrane are marked with arrows.

\section{$\mathrm{CHS}^{-}$and POPG segregate towards scaffold protein}

In Figure 4, two-dimensional maps of thickness, order parameter, and density of nanodiscs, and in Figure 5, profiles of the order parameter for four zones of lipids located at different distances from the nanodisc center are shown. Results obtained for POPC/POPG and POPC/POPG/CHL nanodiscs are in agreement with previously published study of nanodiscs with neutral lipids. ${ }^{36,54,55}$ In these nanodiscs the thickness of bilayer is lowest near the MSPs and gradually increases towards the center of nanodisc. Increase in thickness is associated with increase in density and ordering of lipids. For POPC/POPG/CHSO nanodisc very similar behavior is observed however certain inhomogeneity is seen in the nanodisc. Interestingly for POPC/POPG/CHS ${ }^{-}$nanodisc we observe increase in thickness near the region neighboring MSPs and decrease in the thickness towards the center of nanodisc. Consequently, there were no increase of the order of the tails and density in the center of nanodisc. Moreover, $\mathrm{POPC} / \mathrm{POPG} / \mathrm{CHS}^{-}$nanodisc is also heterogeneous in the central portion. 


\section{Thickness [nm] Order Parameter Mass Density [kg/m3]}

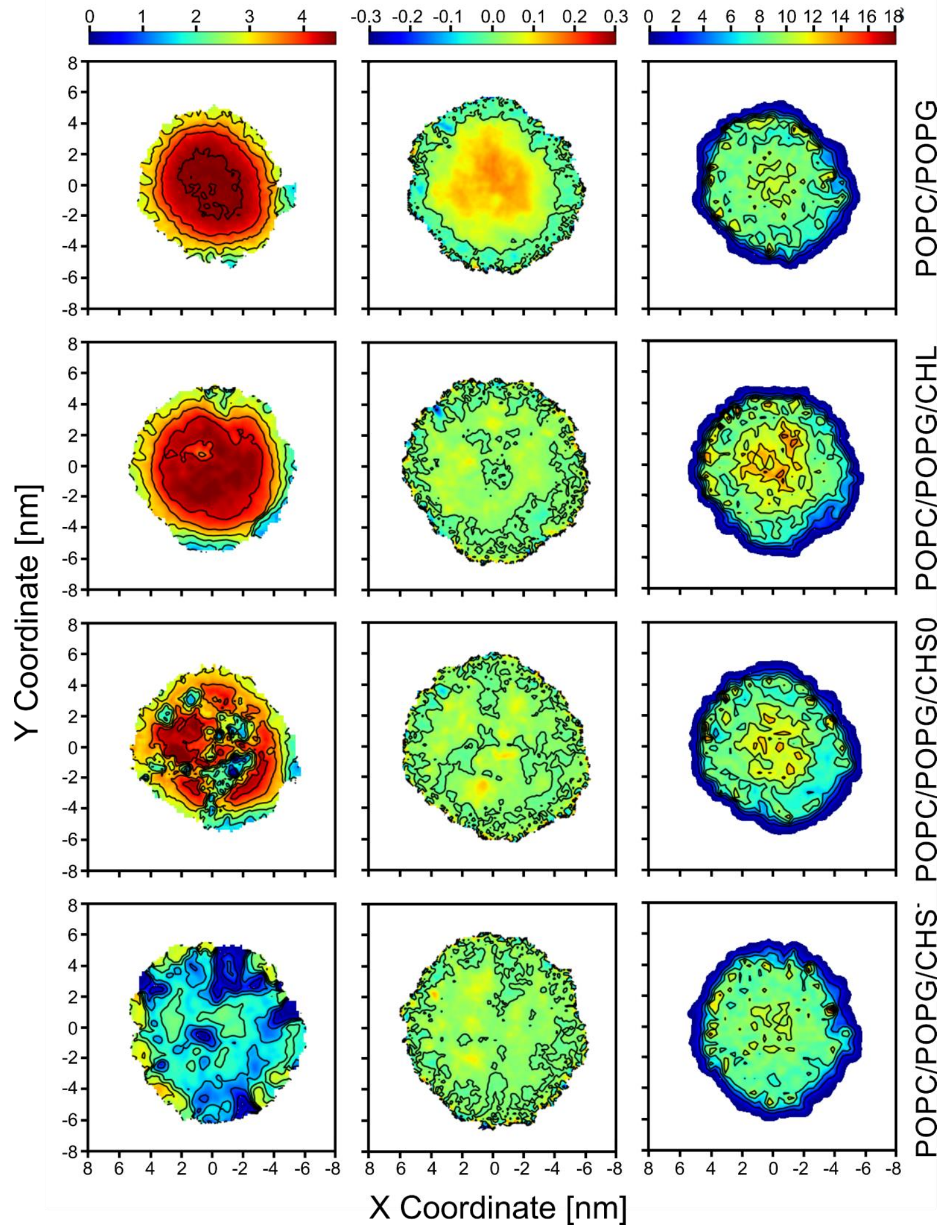

Figure 4. Two-dimensional map showing bilayer thickness (left panel), lipid acyl chain order parameter (central panel), and membrane density map (right panel) for MSP1D1 and nanodiscs at $310 \mathrm{~K}$. 

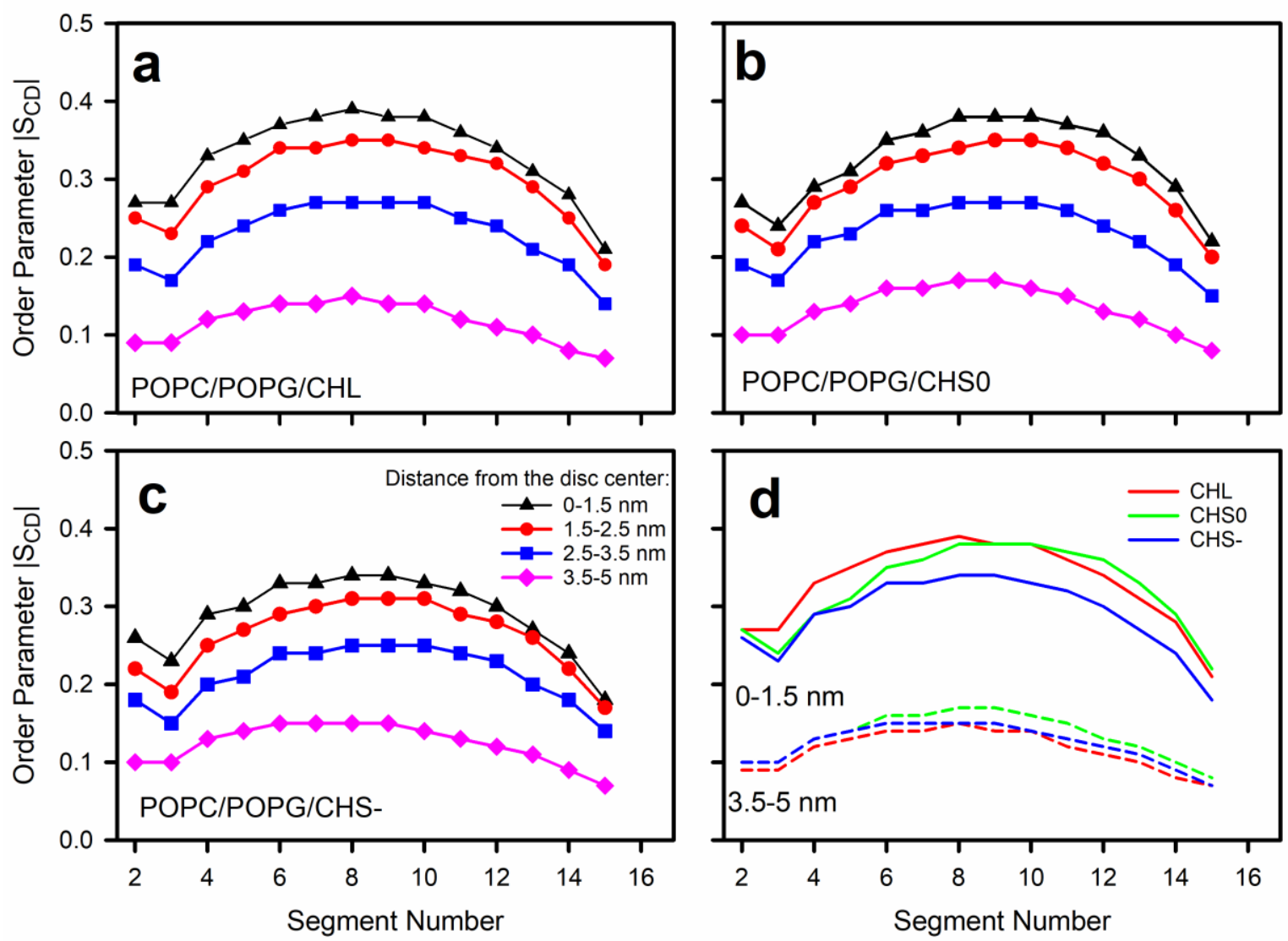

Figure 5. Profiles of the order parameter $S_{C D}$ along hydrocarbon tails of lipids located in four zones: (1) 0-1.5 (black triangles), (2) 1.5-2.5 (read circles), (3) 2.5-3.5 (blue squares), (4) >3.5 (magenta diamonds) nm from the disc center, in POPC/POPG/CHL (a), POPC/POPG/CHSO (b), POPC/POPG/CHS- (c) nanodiscs. Profiles of the order parameter $S_{C D}$ along hydrocarbon tails for lipids located in first zone (solid lines), and fourth zone (dashed lines), in POPC/POPG/CHL (read line), POPC/POPG/CHSO (green line), POPC/POPG/CHS- (blue line) nanodiscs (d).

To understand the distinct lipid properties in POPC/POPG/CHS ${ }^{-}$system, we calculated twodimensional maps showing sterol density (Figure 6) in nanodiscs, and we visualized final location of sterols in the disc (Figure 7). In CHL and CHSO systems, the sterols concentrate mainly in the center of nanodisc, whereas in $\mathrm{CHS}^{-}$system the sterols have clear preferences to localize near the MSPs. Figure 6 showing final snapshots of the MSPs and sterols in nanodiscs further confirms the interaction between MSPs and $\mathrm{CHS}^{-}$. The difference between neutral and ionic form of $\mathrm{CHS}$ indicates that origin of the preference of $\mathrm{CHS}^{-}$towards MSPs is a result of strong electrostatic interactions, salt bridges between carboxylic group of $\mathrm{CHS}^{-}$and 
positively charged amino acids of the protein, predominately arginine (Table 2). These interactions most likely slow the mixing of the lipids in the nanodiscs, thus resulting in the heterogeneity of the lipid properties like, e.g., thickness (Figure 4).

Table 2. Numbers of $\mathrm{H}$-bonds between sterols and MSP and positively charged amino acids of the MSP1: lysine (LYS), arginine (ARG) and histidine (HIS) per sterols molecule.

\begin{tabular}{|l|l|l|l|l|}
\hline & All bonds & LYS - sterol & ARG - sterol & HIS - sterol \\
\hline POPC/POPG/CHL & 0.05 & 0.00 & 0.02 & 0.00 \\
\hline POPC/POPG/CHSO & 0.03 & 0.00 & 0.02 & 0,00 \\
\hline POPC/POPG/CHS- & 0.87 & 0.06 & 0.63 & 0,00 \\
\hline
\end{tabular}

POPG lipid is another anionic molecule in our nanodiscs, therefore we also calculated twodimensional density maps for POPG lipid (Figure 6). Apparently, POPG also accumulates near the MSPs due to strong electrostatic interactions and this is most likely the reason for lack of ordering effect of $\mathrm{CHS}^{-}$on POPG tails.

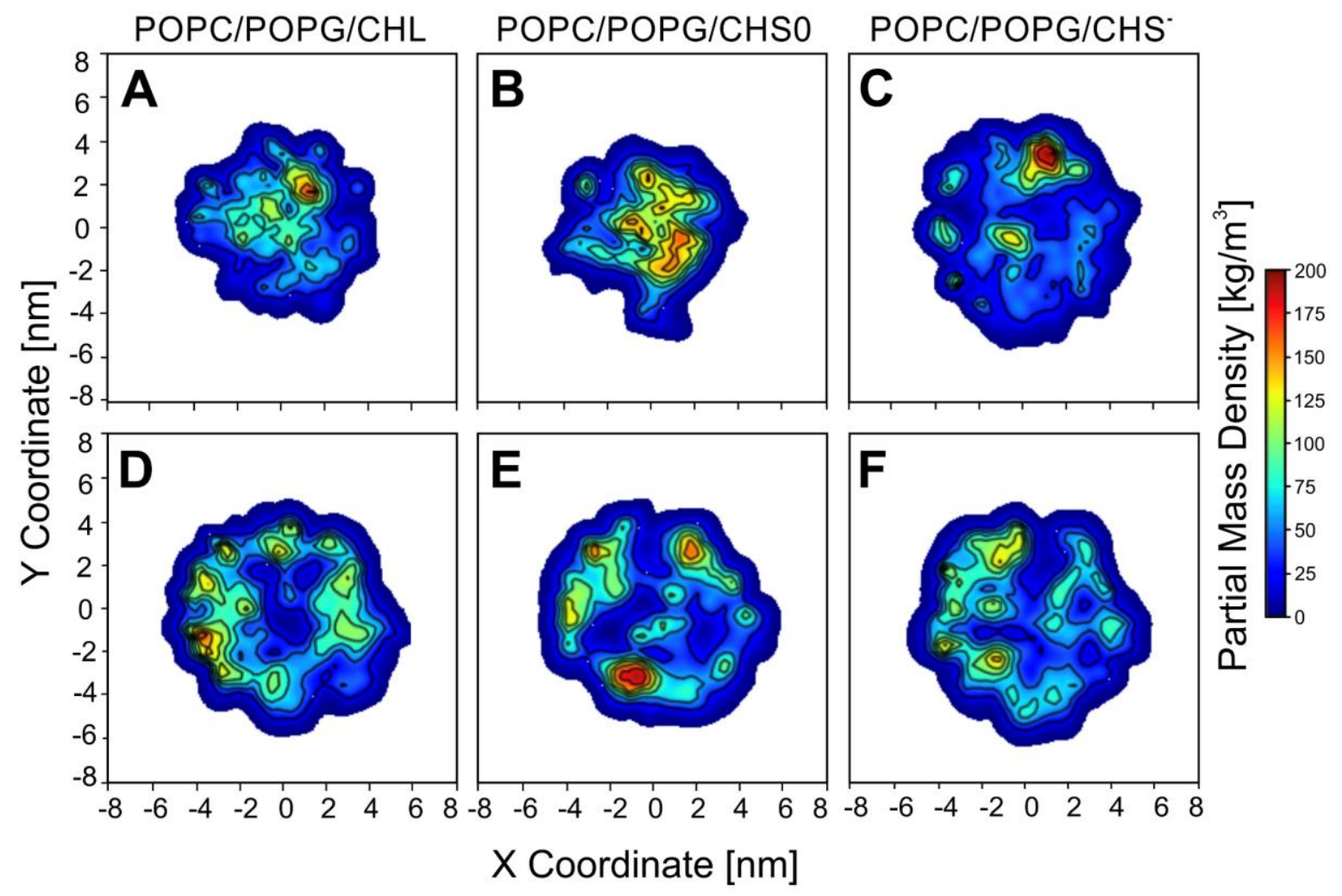

Figure 6. Two-dimensional map showing density of sterols (A, B, and C) and POPG (D, E, and $F)$ in nanodiscs. 

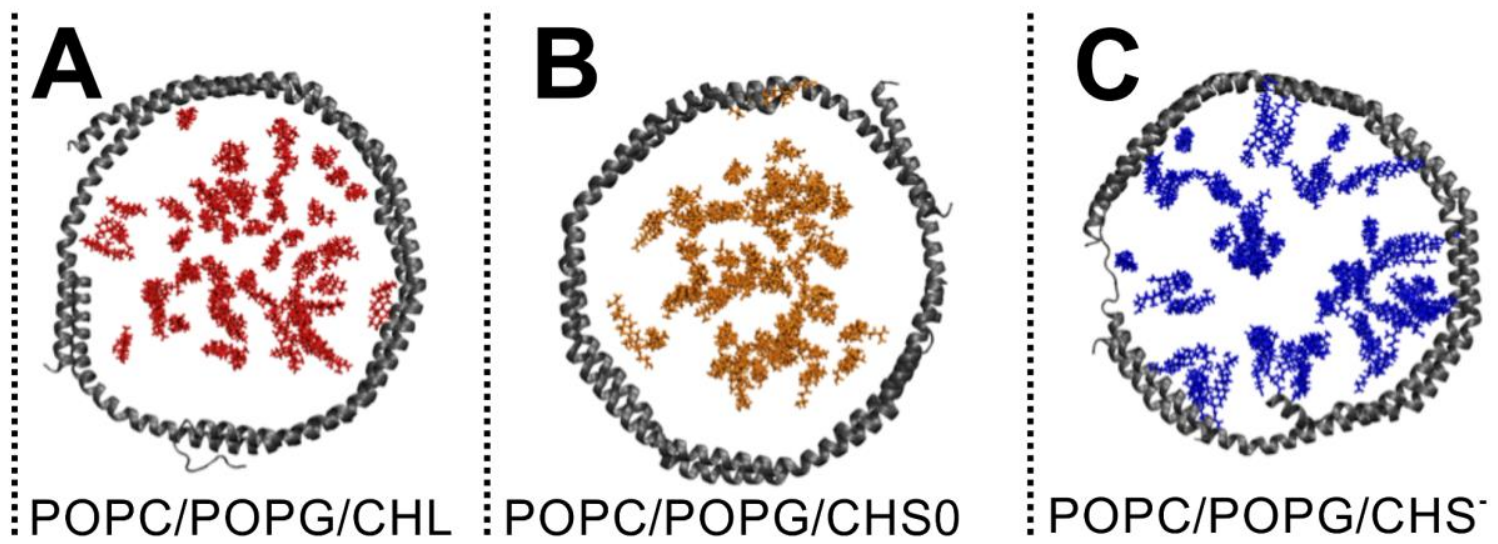

Figure 7. Final snapshots from MD simulations showing locations of sterols in nanodiscs.

\section{CONCLUSIONS}

Although lipid nanodiscs seem to be simple structure, mimicking the lipid environment of MPs and the structure of the nanodiscs is complex. Moreover, the effect of MSPs on lipid behavior is significant. Previous studies have shown that lipids in nanodiscs are more ordered than in liposomes and the lipids close to the MSPs are less ordered than in the center of the nanodisc. In the current study, we show that MSPs affect the distribution of anionic lipids in the nanodisc plane. Both CHS- and POPG preferentially locate close to MSPs due to electrostatic interactions with the positively charged residues of MSPs. These interactions affect the properties of lipids in nanodiscs and are very different in comparison with nanodiscs which contain cholesterol. Particularly striking is the inhomogeneity of nanodiscs with CHS- resulting from slow mixing of lipids in the nanodisc. Our results indicate that CHS is not suitable to mimic CHL.

\section{ACKNOWLEDGEMENTS}

For financial support, we thank the Academy of Finland the Center of Excellence program (grant no. 307415 (PC, TR)). CSC-IT Centre for Science (Espoo, Finland; project number tty3995) and the Finnish Grid and Cloud Infrastructure (persistent identifier urn:nbn:fi:research-infras-2016072533) are acknowledged for excellent computational resources. This work was also supported by Bratniak Foundation of Jagiellonian University which covered partially the cost of BA visiting in Finland. Faculty of Biochemistry, Biophysics and Biotechnology of Jagiellonian University is a partner of the Leading National Research Center (KNOW) supported by the Ministry of Science and Higher Education.

Supporting Information. Package of input files for simulations in GROMACS used in this study. 


\section{References}

(1) Denisov, I. G.; Grinkova, Y. V.; Lazarides, A. A.; Sligar, S. G. Directed Self-Assembly of Monodisperse Phospholipid Bilayer Nanodiscs with Controlled Size. J. Am. Chem. Soc. 2004, 126 (11), 3477-3487.

(2) Bayburt, T. H.; Sligar, S. G. Membrane Protein Assembly into Nanodiscs. FEBS Lett. 2010, 584, 1721-1727.

(3) Huang, R.; Silva, R. A. G. D.; Jerome, W. G.; Kontush, A.; Chapman, M. J.; Curtiss, L. K.; Hodges, T. J.; Davidson, W. S. Apolipoprotein A-I Structural Organization in HighDensity Lipoproteins Isolated from Human Plasma. Nat. Struct. Mol. Biol. 2011, 18 (4), 416-423.

(4) Denisov, I. G.; Sligar, S. G. Nanodiscs for Structural and Functional Studies of Membrane Proteins. Nat. Struct. Mol. Biol. 2016, 23 (6), 481-486.

(5) Dörr, J. M.; Scheidelaar, S.; Koorengevel, M. C.; Dominguez, J. J.; Schäfer, M.; Walree, C. A. van; Killian, J. A. The Styrene-maleic Acid Copolymer: A Versatile Tool in Membrane Research. Eur. Biophys. J. 2016, 45, 3-21.

(6) Wisniewska-Becker, A.; Gruszecki, W. Biomembrane Models. In Woodhead Publishing Series in Biomedicine, Drug-Biomembrane Interaction Studies; Pignatello, R., Ed.; 2013; pp 47-95.

(7) Ritchie, T. K.; Grinkova, Y. V.; Bayburt, T. H.; Denisov, I. G.; Zolnerciks, J. K.; Atkins, W. M.; Sligar, S. G. Chapter 11 Reconstitution of Membrane Proteins in Phospholipid Bilayer Nanodiscs. Methods Enzymol. 2009, 464, 211-231.

(8) Denisov, I. G.; Sligar, S. G. Nanodiscs in Membrane Biochemistry and Biophysics. Chem. Rev. 2017, 117 (6), 4669-4713.

(9) Wadsäter, M.; Maric, S.; Simonsen, J. B.; Mortensen, K.; Cardenas, M. The Effect of Using Binary Mixtures of Zwitterionic and Charged Lipids on Nanodisc Formation and Stability. Soft Matter 2013, 9 (7), 2329-2337.

(10) Lange, Y.; Steck, T. L. Active Membrane Cholesterol as a Physiological Effector. Chem. Phys. Lipids 2016, 199, 74-93.

(11) Epand, R. M.; Chattopadhyay, A. Introduction to the Special Issue on "Properties and Functions of Cholesterol." Chem. Phys. Lipids 2016, 199, 1-2.

(12) Menon, A. K.; Levine, T. P. The Cellular Lipid Landscape. Biochim. Biophys. Acta - Mol. Cell Biol. Lipids 2016, 1861 (8), 755-756.

(13) Yang, Y.; Lee, M.; Fairn, G. D. Phospholipid Subcellular Localization and Dynamics. J. Biol. Chem. 2018, 293 (17), 6230-6240.

(14) Róg, T.; Pasenkiewicz-Gierula, M.; Vattulainen, I.; Karttunen, M. Ordering Effects of Cholesterol and Its Analogues. Biochim. Biophys. Acta - Biomembr. 2009, 1788 (1), 97121.

(15) Róg, T.; Vattulainen, I. Cholesterol, Sphingolipids, and Glycolipids: What Do We Know about Their Role in Raft-like Membranes? Chem. Phys. Lipids 2014, 184, 82-104.

(16) Ohvo-Rekilä, H.; Ohvo-rekila, H.; Ramstedt, B.; Leppima, P.; Slotte, J. P. Cholesterol Interactions with Phospholipids in Membranes. Prog. Lipid Res. 2002, 41 (1), 66-97.

(17) Fantini, J.; Di Scala, C.; Baier, C. J.; Barrantes, F. J. Molecular Mechanisms of ProteinCholesterol Interactions in Plasma Membranes: Functional Distinction between Topological (Tilted) and Consensus (CARC/CRAC) Domains. Chem. Phys. Lipids 2016, 199, 52-60. 
(18) Gimpl, G. Interaction of G Protein Coupled Receptors and Cholesterol. Chem. Phys. Lipids 2016, 199, 61-73.

(19) Howell, S. C.; Mittal, R.; Huang, L.; Travis, B.; Breyer, R. M.; Sanders, C. R. CHOBIMALT: A Cholesterol-Based Detergent. Biochemistry 2011, 49 (44), 9572-9583.

(20) Martinez, D.; Decossas, M.; Kowal, J.; Frey, L.; Stahlberg, H.; Dufourc, E. J.; Riek, R.; Habenstein, B.; Bibow, S.; Loquet, A. Lipid Internal Dynamics Probed in Nanodiscs. ChemPhysChem 2017, 18 (19), 2651-2657.

(21) Wan, C. P. L.; Chiu, M. H.; Wu, X.; Lee, S. K.; Prenner, E. J.; Weers, P. M. M. Apolipoprotein-Induced Conversion of Phosphatidylcholine Bilayer Vesicles into Nanodisks. Biochim. Biophys. Acta - Biomembr. 2011, 1808 (3), 606-613.

(22) Warne, T.; Moukhametzianov, R.; Baker, J. G.; Nehmé, R.; Edwards, P. C.; Leslie, A. G. W.; Schertler, G. F. X.; Tate, C. G. The Structural Basis for Agonist and Partial Agonist Action on a B1-Adrenergic Receptor. Nature 2011, 469, 241-245.

(23) Zocher, M.; Zhang, C.; Rasmussen, S. G. F.; Kobilka, B. K.; Muller, D. J. Cholesterol Increases Kinetic, Energetic, and Mechanical Stability of the Human $\beta_{2}$-Adrenergic Receptor. Proc. Natl. Acad. Sci. 2012, 109 (50), E3463-E3472.

(24) Christopher, J. A.; Brown, J.; Doré, A. S.; Errey, J. C.; Koglin, M.; Marshall, F. H.; Myszka, D. G.; Rich, R. L.; Tate, C. G.; Tehan, B.; et al. Biophysical Fragment Screening of the B1-Adrenergic Receptor: Identification of High Affinity Arylpiperazine Leads Using Structure-Based Drug Design. J. Med. Chem. 2013, 56 (9), 3446-3455.

(25) Enkavi, G.; Javanainen, M.; Kulig, W.; Róg, T.; Vattulainen, I. Multiscale Simulations of Biological Membranes: The Challenge to Understand Biological Phenomena in a Living Substance. Chem. Rev. 2019, 119, 5607-5774.

(26) O’Malley, M. A.; Helgeson, M. E.; Wagner, N. J.; Robinson, A. S. Toward Rational Design of Protein Detergent Complexes: Determinants of Mixed Micelles That Are Critical for the in Vitro Stabilization of a G-Protein Coupled Receptor. Biophys. J. 2011, 101, 1938-1948.

(27) Thompson, A. A.; Liu, J. J.; Chun, E.; Wacker, D.; Wu, H.; Cherezov, V.; Stevens, R. C. GPCR Stabilization Using the Bicelle-like Architecture of Mixed Sterol-Detergent Micelles. Methods 2011, 55 (4), 310-317.

(28) Vukoti, K.; Kimura, T.; Macke, L.; Gawrisch, K.; Yeliseev, A. Stabilization of Functional Recombinant Cannabinoid Receptor CB2 in Detergent Micelles and Lipid Bilayers. PLoS One 2012, 7 (10), e46290.

(29) O'Malley, M. A.; Helgeson, M. E.; Wagner, N. J.; Robinson, A. S. The Morphology and Composition of Cholesterol-Rich Micellar Nanostructures Determine Transmembrane Protein (GPCR) Activity. Biophys. J. 2011, 100 (2), L11-L13.

(30) Oates, J.; Faust, B.; Attrill, H.; Harding, P.; Orwick, M.; Watts, A. The Role of Cholesterol on the Activity and Stability of Neurotensin Receptor 1. Biochim. Biophys. Acta-Biomembr. 2012, 1818 (9), 2228-2233.

(31) Kulig, W.; Tynkkynen, J.; Javanainen, M.; Manna, M.; Róg, T.; Vattulainen, I.; Jungwirth, P. How Well Does Cholesteryl Hemisuccinate Mimic Cholesterol in Saturated Phospholipid Bilayers? J. Mol. Model. 2014, 20, 2121 (1-9).

(32) Kulig, W.; Jurkiewicz, P.; Olzyńska, A.; Tynkkynen, J.; Javanainen, M.; Manna, M.; Róg, T.; Hof, M.; Vattulainen, I.; Jungwirth, P. Experimental Determination and Computational Interpretation of Biophysical Properties of Lipid Bilayers Enriched by Cholesteryl Hemisuccinate. Biochim. Biophys. Acta-Biomembr. 2015, 1848 (2), 422432. 
(33) Hafez, I. M.; Cullis, P. R. Cholesteryl Hemisuccinate Exhibits PH Sensitive Polymorphic Phase Behavior. Biochim. Biophys. Acta-Biomembr. 2000, 1463 (1), 107-114.

(34) Lee, J.; Cheng, X.; Swails, J. M.; Yeom, M. S.; Eastman, P. K.; Lemkul, J. A.; Wei, S.; Buckner, J.; Jeong, J. C.; Qi, Y.; et al. CHARMM-GUI Input Generator for NAMD, GROMACS, AMBER, OpenMM, and CHARMM/OpenMM Simulations Using the CHARMM36 Additive Force Field. J. Chem. Theory Comput. 2016, 12 (1), 405-413.

(35) Jo, S.; Kim, T.; Iyer, V.; Im, W. CHARMM GUI: A Web Based Graphical User Interface for CHARMM. J. Comput. Chem. 2008, 29 (11), 1859-1865.

(36) Qi, Y.; Lee, J.; Klauda, J. B.; Im, W. CHARMM-GUI Nanodisc Builder for Modeling and Simulation of Various Nanodisc Systems. J. Comput. Chem. 2019, 40, 893-899.

(37) MacKerell, A. D.; Bashford, D.; Bellott, M.; Dunbrack, R. L.; Evanseck, J. D.; Field, M. J.; Fischer, S.; Gao, J.; Guo, H.; Ha, S.; et al. All-Atom Empirical Potential for Molecular Modeling and Dynamics Studies of Proteins. J. Phys. Chem. B 1998, 102 (18), 35863616.

(38) Klauda, J. B.; Venable, R. M.; Freites, J. A.; O'Connor, J. W.; Tobias, D. J.; MondragonRamirez, C.; Vorobyov, I.; MacKerell, A. D.; Pastor, R. W. Update of the CHARMM AllAtom Additive Force Field for Lipids: Validation on Six Lipid Types. J. Phys. Chem. $B$ 2010, 114 (23), 7830-7843.

(39) Essmann, U.; Perera, L.; Berkowitz, M. L.; Darden, T.; Lee, H.; Pedersen, L. G. A Smooth Particle Mesh Ewald Method. J. Chem. Phys. 1995, 103 (19), 8577-8593.

(40) Hess, B.; Bekker, H.; Berendsen, H. J. C.; Fraaije, J. G. E. M. LINCS: A Linear Constraint Solver for Molecular Simulations. J. Comput. Chem. 1997, 18 (12), 1463-1472.

(41) Nosé, S. A Unified Formulation of the Constant Temperature Molecular Dynamics Methods. J. Chem. Phys. 1984, 81 (1), 511-519.

(42) Hoover, W. G. Canonical Dynamics: Equilibrium Phase-Space Distributions. Phys. Rev. A 1985, 31 (3), 1695-1697.

(43) Parrinello, M.; Rahman, A. Polymorphic Transitions in Single Crystals: A New Molecular Dynamics Method. J. Appl. Phys. 1981, 52 (12), 7182-7190.

(44) Abraham, M. J.; Murtola, T.; Schulz, R.; Páll, S.; Smith, J. C.; Hess, B.; Lindah, E. Gromacs: High Performance Molecular Simulations through Multi-Level Parallelism from Laptops to Supercomputers. SoftwareX 2015, 1-2, 19-25.

(45) Castillo, N.; Monticelli, L.; Barnoud, J.; Tieleman, D. P. Free Energy of WALP23 Dimer Association in DMPC, DPPC, and DOPC Bilayers. Chem. Phys. Lipids 2013, 169, 95-105.

(46) Humphrey, W.; Dalke, A.; Schulten, K. VMD-Visual Molecular Dynamics. J. Mol. Graph. 1996, 14, 33-38.

(47) Denisov, I. G.; Baas, B. J.; Grinkova, Y. V.; Sligar, S. G. Cooperativity in Cytochrome P450 3A4: Linkages in Substrate Binding, Spin State, Uncoupling, and Product Formation. J. Biol. Chem. 2007, 282, 7066-7076.

(48) Stepien, P.; Polit, A.; Wisniewska-Becker, A. Comparative EPR Studies on Lipid Bilayer Properties in Nanodiscs and Liposomes. Biochim. Biophys. Acta-Biomembr. 2015, 1848 (1), 60-66.

(49) Budil, D. E.; Sanghyuk, L.; Saxena, S.; Freed, J. H. Nonlinear-Least-Squares Analysis of Slow-Motion EPR Spectra in One and Two Dimensions Using a Modified LevenbergMarquardt Algorithm. J. Magn. Reson. - Ser. A 1996, 183 (1), 152-159.

(50) Schneider, DJ.; Freed, J. Calculating Slow Motional Magnetic Resonance Spectra: A User's Guide. In Spin Labeling: Theory and Application; New York, 1989; pp 1-76.

(51) M, G.; JH, F. Hydration, Structure and Molecular Interactions in the Headgroup Region 
of Dioleoylphosphatidylcholine Bilayers: An Electron Spin Resonance Study. Biophys. J. 2003, 85, 4023-4040.

(52) Meirovitch, E.; Freed, J. H. Analysis of Slow-Motional Electron Spin Resonance Spectra in Smectic Phases in Terms of Molecular Configuration, Intermolecular Interactions, and Dynamics. J. Phys. Chem. 1984, 88, 4995-5004.

(53) Kusumi, A.; Pasenkiewicz-Gierula, M. Rotational Diffusion of a Steroid Molecule in Phosphatidylcholine Membranes: Effects of Alkyl Chain Length, Unsaturation, and Cholesterol as Studied by a Spin-Label Method. Biochemistry 1988, 27, 4407-4415.

(54) Pourmousa, M.; Pastor, R. W. Molecular Dynamics Simulations of Lipid Nanodiscs. Biochim. Biophys. Acta - Biomembr. 2018, 1860 (10), 2094-2107.

(55) Siuda, I.; Tieleman, D. P. Molecular Models of Nanodiscs. J. Chem. Theory Comput. 2015, 11 (10), 4923-4932. 
TOC Graphic

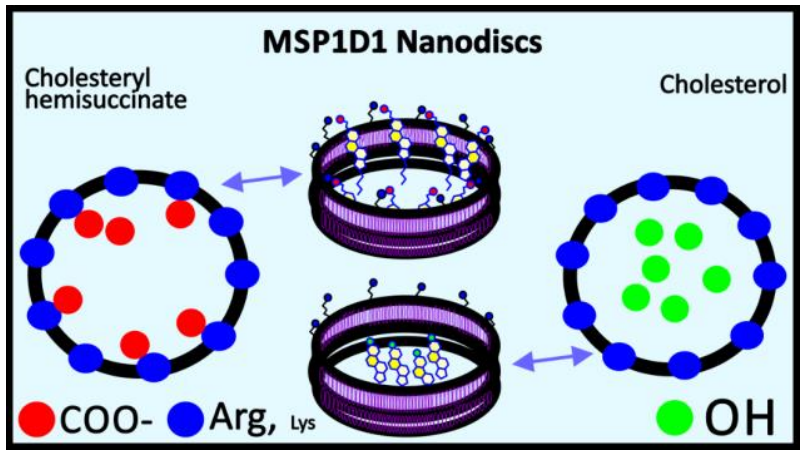

